\title{
Leptomycin B, a metabolite of Streptomyces, inhibits the expression of inducible nitric oxide synthase in BV2 microglial cells
}

\author{
BYEONG-CHURL JANG ${ }^{1}$, SU-HAENG SUNG ${ }^{1}$, JONG-GU PARK ${ }^{1}$, JONG-WOOK PARK ${ }^{1}$, MIN-HO SUH $^{1}$, \\ IN-HAK CHOI ${ }^{2}$, MINORU YOSHIDA $^{3}$, SUN-KYUN YOO ${ }^{4}$ and SEONG-IL SUH ${ }^{1}$
}

\begin{abstract}
${ }^{1}$ Chronic Disease Research (CDR) Center \& Institute for Medical Science, Keimyung University School of Medicine, \#194 DongSan-Dong, Jung-Gu, Daegu 700-712; ${ }^{2}$ Department of Microbiology, Inje University College of Medicine, \#633-165 GaeKeum-Dong, Jin-Gu, Busan 614-735, South Korea; ${ }^{3}$ Chemical Genetics Laboratory, RIKEN, Hirosawa 2-1, Wako, Saitama 351-0198, Japan; ${ }^{4}$ Department of Oriental Medicine and Food Biotechnology, Joongbu University, Kumsan-gun, Chungnam 312-702, South Korea
\end{abstract}

Received April 14, 2006; Accepted June 6, 2006

\begin{abstract}
Overexpression of inducible nitric oxide synthase (iNOS) and the resultant overproduction of NO has been implicated in neuronal inflammatory diseases. Leptomycin B (LMB), a metabolite of Streptomyces, has been identified as a specific inhibitor of CRM1 nuclear export receptor. In this study, we evaluated the effect of LMB on lipopolysaccharide (LPS)-induced iNOS expression in BV2 cells, a murine microglial cells and the associated mechanisms. LMB strongly inhibited LPS-induced iNOS protein and mRNA expressions in BV2 cells in which $10 \mathrm{ng} / \mathrm{ml}$ of LMB $(18 \mathrm{nM})$ was sufficient to greatly down-regulate iNOS by LPS, suggesting the potency of LMB to inhibit iNOS. The data of iNOS promoter-driven luciferase assay further suggested that the LMB inhibitory effect was in part due to inhibition of iNOS transcription. However, LPS-induced activation of various intracellular signaling proteins, such as nuclear factor- $\kappa \mathrm{B}(\mathrm{NF}-\kappa \mathrm{B})$, extracellular signal-regulated kinases (ERKs), p38s, and c-Jun N-terminal kinases (JNKs), whose activations are known to be important for iNOS expression by LPS in BV2 cells, were not affected in the presence of LMB. Together, these results suggest that LMB inhibits iNOS expression in response to LPS in BV2 microglia, and the inhibition seems to be associated with blockage of CRM1mediated iNOS mRNA nuclear export and also in part transcriptional down-regulation of iNOS, but not through
\end{abstract}

Correspondence to: Dr Byeong-Churl Jang and Dr Seong-Il Suh, Chronic Disease Research Center and Institute for Medical Science, Keimyung University School of Medicine, \#194 DongSan-Dong, Jung-Gu, Daegu 700-712, South Korea

E-mail: jangbc12@kmu.ac.kr seong@dsmc.or.kr

Key words: Leptomycin B, iNOS, LPS, mRNA stability and nuclear export, transcription, BV2 cells modulation of NF-кB and the mitogen-activated protein kinase signaling pathways.

\section{Introduction}

Nitric oxide (NO) is an important signaling molecule involved in vascular homeostasis, neurotransmission, and immune defense against infectious agents (1). Importantly, recent studies have demonstrated that the expression and activity of iNOS, one enzyme producing $\mathrm{NO}$, are abnormally increased in inflammation and cancer, and the resultant $\mathrm{NO}$ overproduction plays a causative role in these processes $(2,3)$.

NO is produced from L-arginine and molecular oxygen by the action of NOS $(4,5)$. Three isoforms of NOS (type I, II, and III) have been molecularly identified and cloned in mammals (4-6). The neuronal (type I, nNOS) and endothelial (type III, eNOS) enzymes are constitutively expressed and thought to involve in physiological functions in neuronal and endothelial systems, respectively. On the other hand, the third member of NOS family is an inducible enzyme (iNOS, type II). It has been shown that iNOS expression is largely increased in a variety of cells after the exposure of LPS and interferon- $\gamma$ (IFN- $\gamma$ ) (7), cytokines (8), or catalase (9).

Regulation of iNOS expression is complex. Primarily, it is regulated at transcription $(4,5,10)$. iNOS transcription in response to LPS or cytokines is greatly dependent on activities of transcription factors, including nuclear factor- $\mathrm{B}(\mathrm{NF}-\kappa \mathrm{B})$, activator protein-1, nuclear factor-interleukin 6 (NF-IL6), or IFN- $\gamma$ responsive factor, which individually acts on its cognate cis-acting element within iNOS promoter (11-13). iNOS expression can also be regulated at mRNA stability or translation (14-16). Furthermore, it is well established that iNOS expression depends on the activity of intracellular signaling proteins, including p38s, c-Jun N-terminal kinases (JNKs), extracellular signal-regulated kinases (ERKs), phosphatidylinositol 3-kinase (PI3K)/AKT, and p70S6K, which affects iNOS transcription, mRNA stability, and/or protein synthesis (17-20). 
Leptomycin B (LMB) was originally discovered as a potent anti-fungal antibiotic from Streptomyces species (21). The cellular target of LMB has been identified as the nuclear export receptor CRM1/exportin 1 (22), which is involved in nuclear trafficking of cellular RNAs or proteins containing the nuclear export sequence (NES) (23). Supporting this, LMB is shown to inhibit nuclear export of RNAs such as cfos (24) or of the NES-containing proteins such as $\alpha$-catenin (25) or inhibitor of $\kappa B$-alpha $($ I $\kappa$ B- $\alpha$ ) (26). Of interest, LMB was shown to inhibit mRNA nuclear export of cyclooxygenase-2 (COX-2), thus down-regulating the expression of COX-2, an inflammatory enzyme producing prostaglandins, in MDA-MB-231 cells (27).

In this study, we evaluated the effect of LMB on the regulation of iNOS expression in response to LPS, a wellknown iNOS inducer, in BV2 cells, a murine microglial cell line and possible inhibitory mechanisms by which LMB regulates iNOS expression.

\section{Materials and methods}

Materials. The anti-actin antibody, aprotinin, leupeptin, phenanthroline, and benzamidine- $\mathrm{HCl}$ were purchased from Sigma (St. Louis, MO). Anti-rabbit or mouse secondary horseradish peroxidase antibodies and ECL Western detection reagents were bought from Amersham Biosciences (Amersham, UK). Bradford reagent was from Bio-Rad (Hercules, CA). An anti-iNOS polyclonal antibody was purchased from Cayman Chemicals (Ann Arbor, MI). Antibodies of phospho-ERKs (p-ERKs), phospho-JNKs (pJNKs), phospho-p38s (p-p38s) were obtained from Cell Signaling Tech. (Beverly, MA). The anti-IкB- $\alpha$ antibody was from Santa Cruz Company (Santa Cruz, CA). RPMI-1640, Dulbecco's modified Eagle's medium (DMEM), and mixtures of penicillin/streptomycin were purchased from Gibco-BRL (Gaithersburg, MD).

Cell culture and preparation of whole cell lysates. BV2 murine microglia were cultured in DMEM supplemented with $10 \%$ heat-inactivated fetal bovine serum (FBS), $100 \mathrm{U} / \mathrm{ml}$ penicillin, and $100 \mu \mathrm{g} / \mathrm{ml}$ streptomycin. Raw 264.7 murine macrophages were grown in RPMI supplemented with $10 \%$ FBS, $100 \mathrm{U} / \mathrm{ml}$ penicillin, and $100 \mu \mathrm{g} / \mathrm{ml}$ streptomycin. To prepare whole cell lysates, after stimulation with agents, cells were washed with ice-cold phosphate-buffered saline (PBS) containing $1 \mathrm{mM} \mathrm{Na}_{3} \mathrm{VO}_{4}$ and $1 \mathrm{mM} \mathrm{NaF}$, and lysed in a buffer [50 mM Tris-Cl (pH 7.4), $150 \mathrm{mM} \mathrm{NaCl}, 1 \%$ Triton $\mathrm{X}-100,1 \%$ sodium dodecyl sulfate (SDS), $1 \%$ Nonidet P-40 (NP40), 1 mM EDTA, $200 \mathrm{nM}$ aprotinin, $20 \mu \mathrm{M}$ leupeptin, $50 \mu \mathrm{M}$ phenanthroline, $280 \mu \mathrm{M}$ benzamidine-HCl]. After centrifugation at 12,000 rpm for $20 \mathrm{~min}$ at $4^{\circ} \mathrm{C}$, the supernatant was collected, and the protein concentration was determined.

Western blot analysis. Equal amounts of protein (40 $\mu \mathrm{g} / \mathrm{lane})$ were resolved by $10-12 \%$ SDS-polyacrylamide gel electrophoresis (PAGE), and transferred onto a nitrocellulose membrane (Millipore, Bedford, MA). The membrane was then washed with Tris-buffered saline (TBS, $10 \mathrm{mM}$ Tris, $150 \mathrm{mM} \mathrm{NaCl}$ ) containing $0.05 \%$ Tween-20 (TBST) and blocked in TBST containing 5\% non-fat dried milk. The membrane was further incubated with respective specific antibodies such as iNOS $(1: 1,000)$, p-ERKs $(1: 1,000)$, p-JNKs $(1: 1,000)$, p-p38s $(1: 1,000)$, and actin $(1: 5,000)$. The membrane was continuously incubated with appropriate secondary antibodies coupled to horseradish peroxidase, and developed in the ECL western detection reagents.

iNOS promoter/luciferase assay. Briefly, each $1 \mu \mathrm{g}$ of murine iNOS promoter/luciferase DNA [kindly provided by Dr C.J. Lowenstein (Johns Hopkins University, MD)] along with 20 ng of control pRL-TK DNA (Promega, Madison, WI) was transiently transfected into $1.5 \times 10^{6} \mathrm{BV} 2$ cells/well in 6-well plates using Lipofectamine/Plus reagents (Invitrogen, Carlsbad, CA) according to the manufacturer's instructions. After $24 \mathrm{~h}$ post-transfection, cells were treated with LPS $(1 \mu \mathrm{g} / \mathrm{ml})$ in the absence or presence of $\operatorname{LMB}(1,5$, or $10 \mathrm{ng} / \mathrm{ml})$ for additional $4 \mathrm{~h}$. Cells were then washed, lysed, followed measurement of luciferase activity using a luciferase assay kit (Promega). The luciferase activity was normalized with expression of control pRL-TK.

Reverse transcription-polymerase chain reaction ( $R T-P C R)$. Total RNA was isolated with the RNAzol-B (Tel-Test, Friendswood, TX) according to the instructions provided by the manufacturer. Three micrograms of total RNA were reverse transcribed using M-MLV reverse transcriptase (Promega) following the manufacturer' instructions. Single stranded cDNA was then amplified by PCR with specific primers of iNOS and glyceraldehyde 3-phosphate dehydrogenase (GAPDH): iNOS sense, 5'-GACAAGCTGCATGTG ACATC-3'; iNOS anti-sense, 5'-GCTGGTAGGTTCCT GTTGTT-3'; GAPDH sense, 5'-GGTGAAGGTCGGTGTGA ACG-3'; GAPDH anti-sense, 5'-GGTAGGAACACGGAAG GCCA-3'. The following PCR conditions were applied: iNOS, 25 cycles of denaturation at $94^{\circ} \mathrm{C}$ for $30 \mathrm{sec}$, annealing at $52^{\circ} \mathrm{C}$ for $30 \mathrm{sec}$, and extension at $72^{\circ} \mathrm{C}$ for $30 \mathrm{sec}$; GAPDH, 18 cycles of denaturation at $94^{\circ} \mathrm{C}$ for $30 \mathrm{sec}$, annealing at $57^{\circ} \mathrm{C}$ for $30 \mathrm{sec}$, and extension at $72^{\circ} \mathrm{C}$ for $30 \mathrm{sec}$. The GAPDH was used as an internal control to evaluate relative expression of iNOS.

Subcellular RNA fractionation. Cells were washed in PBS and successively extracted as previously described (27). Briefly, cells were primarily extracted with buffer A [10 mM Tris-Cl (pH 7.5), $10 \mathrm{mM} \mathrm{NaCl}, 3 \mathrm{mM} \mathrm{MgCl}_{2}, 0.5 \%$ (v/v) Nonidet P-40, $40 \mathrm{U} / \mathrm{ml}$ RNasin, $1 \mathrm{mM}$ DTT). The remaining pellet [rough endoplasmic reticulum (RER) and nucleus] was washed in buffer A and extracted in buffer B [10 mM Tris-Cl ( $\mathrm{pH} 7.5), 10 \mathrm{mM} \mathrm{NaCl}, 0.5 \%$ (v/v) Nonidet P-40, $40 \mathrm{mM}$ EDTA, $40 \mathrm{U} / \mathrm{ml}$ RNasin, $1 \mathrm{mM}$ DTT]. The remaining nuclear pellet was washed in buffer $\mathrm{B}$ and extracted with buffer $\mathrm{C}$ [10 mM Tris-Cl (pH 7.5), 0.5 M NaCl, $3 \mathrm{mM} \mathrm{MgCl}_{2}, 0.5 \%$ (v/v) Nonidet P-40, $40 \mathrm{U} / \mathrm{ml}$ RNasin, 1 mM DTT]. RNA was then purified from each fraction by the RNAzol-B.

\section{Results}

Effects of LMB on iNOS protein and $m R N A$ expressions in $L P S$-stimulated BV2 microglia. Initially, we measured the effect of LMB in different concentrations on the expression 


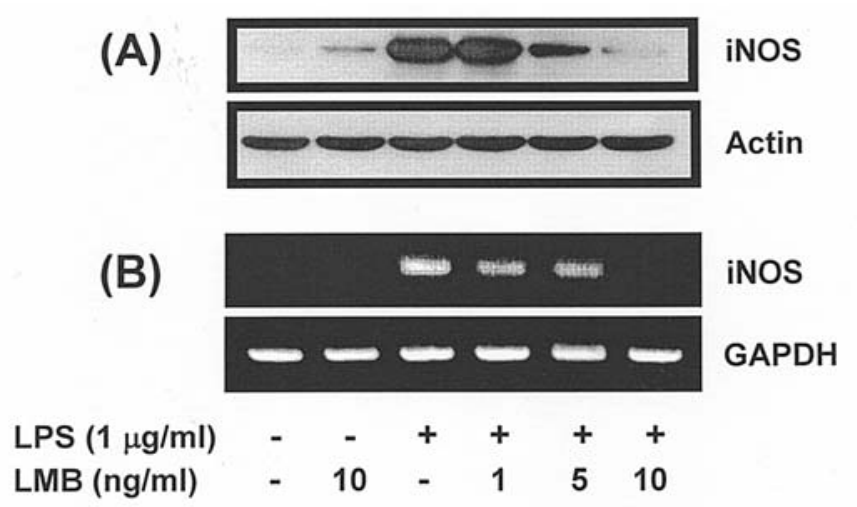

Figure 1. Inhibitory effect of LMB on LPS-induced iNOS expression in BV2 microglia. BV2 cells were pre-treated with the indicated concentrations of LMB for $1 \mathrm{~h}$. Cells were then exposed to LPS for $6 \mathrm{~h}$ in the absence or presence of LMB with the indicated concentrations. Total cell lysates and RNA were prepared, and analyzed for iNOS or actin protein by immunoblotting (A) and iNOS or GAPDH mRNA by RT-PCR (B), respectively.

of iNOS protein and mRNA in LPS-stimulated BV2 cells. As shown in Fig. 1, results of RT-PCR and Western analysis demonstrated that LMB concentration-dependently inhibited LPS-induced expression of iNOS protein (Fig. 1A) and iNOS mRNA (Fig. 1B) in BV2 cells. Interestingly, $10 \mathrm{ng} / \mathrm{ml}$ of LMB (18 nM) was sufficient to greatly down-regulate the LPS-induced iNOS protein and mRNA expressions, suggesting the potency of LMB in down-regulating iNOS. Collectively, these results demonstrate that LMB potently and strongly inhibits the expression of iNOS protein and mRNA by LPS in BV2 microglia.

Effects of LMB on iNOS transcription and iNOS mRNA stability in LPS-stimulated BV2 microglia. It has been well documented that induction of iNOS by extracellular stimuli such as LPS or cytokines correlates with increase in iNOS transcription and mRNA stability (11-13). This led us to test the effect of LMB on iNOS transcription and iNOS mRNA stability in LPS-stimulated BV2 cells using luciferase transfection assay with iNOS promoter/luciferase DNA constructs and actinomycin D chase experiments, respectively. As shown in Fig. 2A, LMB concentration-dependently reduced the expression of luciferase in LPS-stimulated BV2 cells. Treatment of LMB at $10 \mathrm{ng} / \mathrm{ml}$ concentration was found to inhibit about $50 \%$ iNOS transcription in LPS-stimulated BV2 cells. Data of actinomycin D chase experiments also demonstrated that presence of LMB accelerated the destabilization of iNOS mRNA in LPS-stimulated BV2 cells. Taken together, these data suggest that LMB may act at the levels of iNOS transcription and iNOS mRNA stability in response to LPS in BV2 cells.

Effects of LMB on nuclear export of iNOS mRNA in LPSstimulated in BV2 microglia. LMB is a specific inhibitor of CRM1 (22), which mediates the delivery of cellular proteins or RNAs from the nucleus to the cytoplasm (23). Given that LMB inhibits LPS-induced iNOS expression in BV2 cells (Fig. 1), we hypothesized that in BV2 cells LPS might induce CRM1-dependent nuclear export of iNOS mRNA. Thus, we tested if it was inhibited by LMB. BV2 cells were initially
(A)

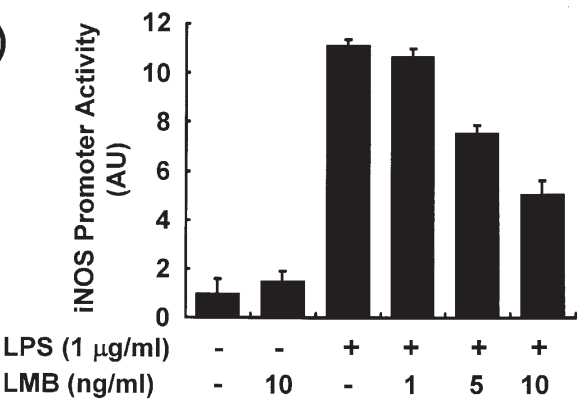

(B)

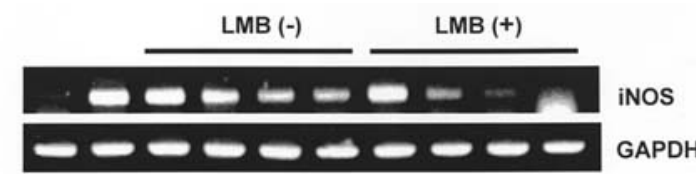

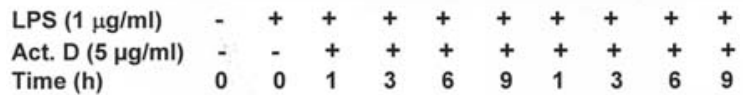

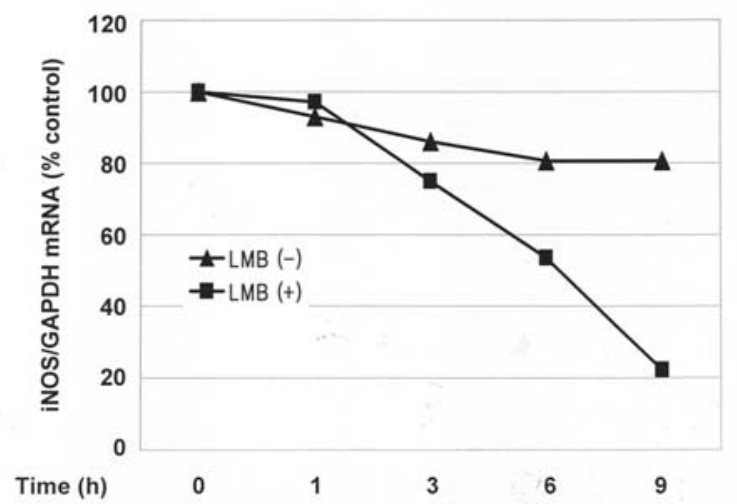

Figure 2. Effects of LMB on iNOS transcription and iNOS mRNA stability in LPS-stimulated BV2 microglia. BV2 cells were co-transfected with $1 \mu \mathrm{g}$ of the murine iNOS promoter-containing luciferase DNA along with $20 \mathrm{ng}$ of control pRL-TK DNA for $24 \mathrm{~h}$ (A). Transfected cells were then treated with LPS in the absence or presence of LMB with the indicated concentrations for $4 \mathrm{~h}$. Cell lysates were prepared, and assayed for reporter gene activity. Data are mean \pm SE of three independent experiments. BV2 cells were initially treated without or with LPS for $4 \mathrm{~h}$ to highly induce iNOS mRNA (B). After $4 \mathrm{~h}$, the conditioned media were removed and cells were further grown without or with LPS along with or without LMB $(10 \mathrm{ng} / \mathrm{ml})$ in the presence of actinomycin D $(5 \mu \mathrm{g} / \mathrm{ml})$ that inhibits ongoing transcription. At each time, total RNA was prepared, and used for iNOS or GAPDH RT-PCR.

pre-treated with LMB for $1 \mathrm{~h}$ and then exposed to LPS for $4 \mathrm{~h}$. Cytoplasmic and nuclear RNA pools were prepared and used for RT-PCR analysis to measure subcellular distribution of iNOS mRNA. Unfortunately, we were not able to measure iNOS mRNA levels under these experimental conditions, because of a very low expression of iNOS mRNA in either total or subcellular RNA pools (data not shown). This is explained with the data of Fig. 1B wherein LMB pre-treatment almost completely inhibited iNOS mRNA expression by LPS, which might be correlated with iNOS transcriptional suppression by LMB as shown in Fig. 2A. This led us to perform an alternative experiment in which BV2 cells were primarily treated with LPS for $4 \mathrm{~h}$ to highly induce iNOS mRNA levels, following 4-h post-treatment with LMB and preparation of subcellular RNA fractions. Under this condition, in BV2 cells, LPS induced high expression of total iNOS mRNA (Fig. 3A, lane 3), cytoplasmic localization of a majority 


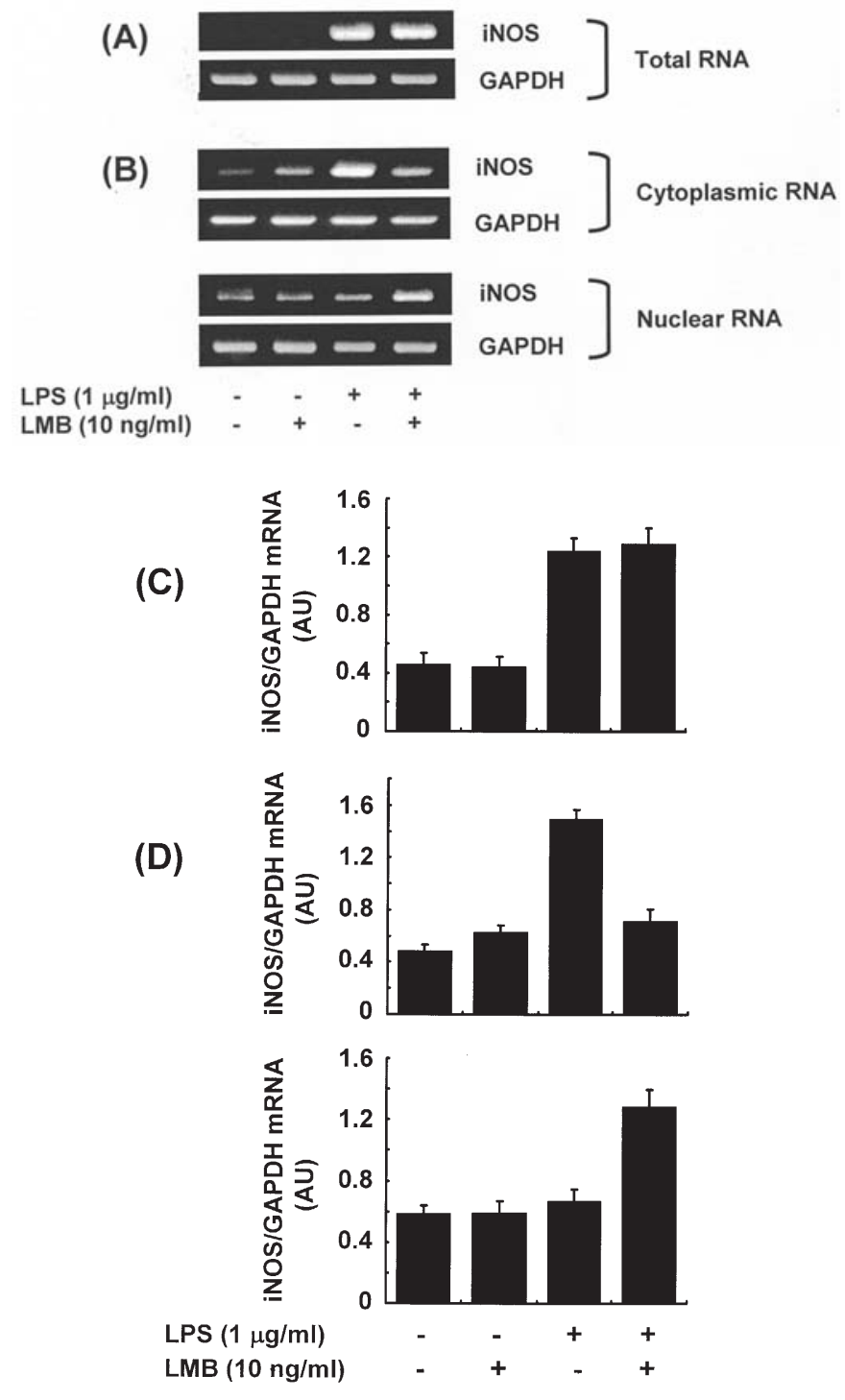

Figure 3. LMB blocks CRM1-mediated nuclear export of iNOS mRNA. BV2 cells were primarily treated with LPS for $4 \mathrm{~h}$ to highly induce iNOS mRNA and then exposed to LMB for additional $4 \mathrm{~h}$. Total RNA was then isolated, and used for iNOS or GAPDH RT-PCR analysis to measure iNOS or GAPDH mRNA levels (A). Subcellular (cytoplasmic and nuclear) RNA was prepared as explained in the Materials and methods (B). The RNA sample fractionated was used for iNOS or GAPDH RT-PCR analysis to measure iNOS or GAPDH mRNA levels. Data are from a representative experiment that was repeated twice. The graph (C) shows iNOS mRNA levels normalized to GAPDH mRNA levels from (A), which is quantitated from two separate experiments. The graph (D) shows iNOS mRNA levels normalized to GAPDH mRNA levels from (B), which is quantitated from two separate experiments. AU, arbitrary unit.

of iNOS mRNA in BV2 cells (Fig. 3B, lane 3), suggesting that iNOS mRNA transcribed following LPS stimulation is exported from the nucleus to the cytoplasm where translation occurs. Unlike the inhibitory effect of LMB pre-treatment on iNOS mRNA expression by LPS (Fig. 1B), however, 4-h post-treatment of LMB did not affect total amounts of iNOS mRNA that was induced by 4-h LPS treatment (Fig. 3A, lane 4). Of interest, LPS-induced subcellular distribution of iNOS mRNA was changed by 4 -h post-treatment of LMB, which effectively blocked LPS-induced cytoplasmic localization of iNOS mRNA, retaining a majority of iNOS
(A)

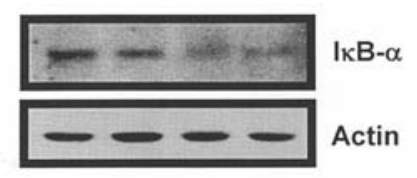

(B)
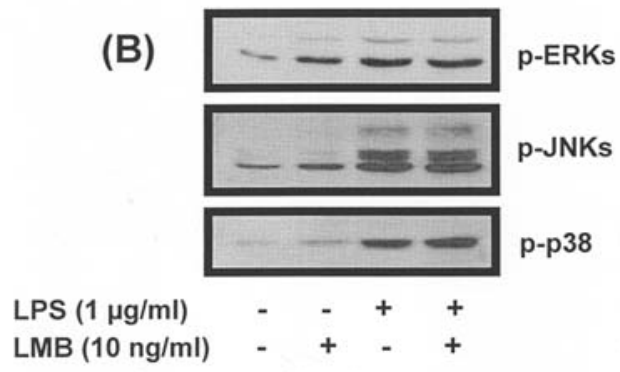

Figure 4. Effects of LMB on LPS-induced activation of MAPKs or NF-кB in BV2 microglia. BV2 cells were pre-treated with LMB for $1 \mathrm{~h}$. Cells were then exposed to LPS for $0.5 \mathrm{~h}$ in the absence or presence of LMB. Total cell lysates were prepared, and used for the measurement of the degree of IкB- $\alpha$ protein degradation (A), an indicator of $\mathrm{NF}-\mathrm{\kappa B}$ activation, or of the extent of phosphorylation of ERKs, JNKs, or p38s (B), an indicator of each protein activation, in response to LPS.

mRNA in the nuclear compartment (Fig. 3B, lane 4). Under same experimental conditions, cellular distribution of GAPDH mRNA was not affected by 4-h post-treatment of LMB, suggesting the specificity of LMB to inhibit nuclear export of iNOS mRNA in response to LPS. Collectively, these results suggest that LMB may inhibit LPS-induced iNOS expression in BV2 cells, in part, by blockage of nuclear export of iNOS mRNA which may occur in a CRM1-dependent pathway.

Effects of $L M B$ on activation of MAPKs and NF- $\mathrm{KB}$ in LPSstimulated $B V 2$ microglia. It is well known that LPS induces activation of various intracellular signaling proteins including NF-кB transcription factor, ERKs, JNKs, and p38 MAPK whose activation is critical for LPS-induced iNOS expression (17-20). This led us to test the effect of LMB on LPS-triggered activation of these signaling pathways. In this study, the degree of phosphorylation of MAPKs (ERKs, JNKs, and p38 MAPK) and of IкB- $\alpha$ degradation, respectively, in response to LPS stimulation was assessed as an indicator of the activation of each MAPK and NF-кB. As anticipated, LPS treatment led to activation of ERKs, JNKs, and p38 MAPK and proteolytic degradation of IкB- $\alpha$ in BV2 cells (Fig. 4A and B, lane 3). Notably, LMB treatment had no effect on LPS-induced activation of ERKs, JNKs, and p38 MAPK and proteolytic degradation of IкB- $\alpha$ in BV2 cells (Fig. 4A and B, lane 4). These results indicate that LMB exert its inhibitory effect on LPS-induced iNOS expression in BV2 cells, not by ERKs, JNKs, p38 MAPK, and NF-кB signaling pathways activated by LPS.

Effects of LMB on iNOS expression by other iNOS inducer or in other type of cells. We further evaluated the effect of LMB on iNOS expression by LPS in Raw 264.7 murine macrophages (Raw 264.7 cells). As shown in Fig. 5A, LMB concentration-dependently inhibited the expression of iNOS by LPS in Raw 264.7 cells. In particular, $2.5 \mathrm{ng} / \mathrm{ml}$ of LMB 

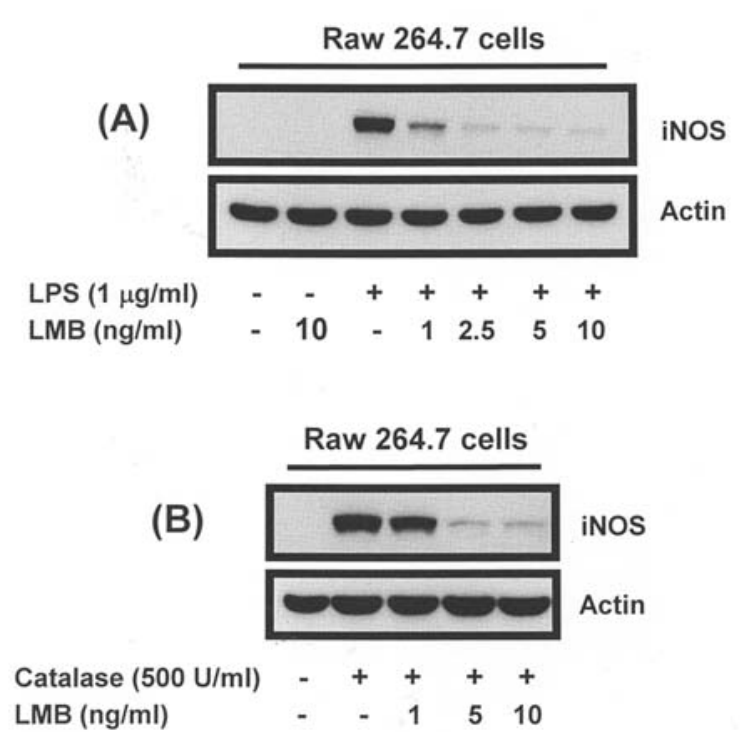

Figure 5. Inhibitory effect of LMB on LPS- or catalase-induced iNOS expression in Raw 264.7 macrophages. Raw 264.7 cells were pre-treated with LMB in the indicated concentrations for $1 \mathrm{~h}$. Cells were then exposed to LPS for $6 \mathrm{~h}$ in the absence or presence of LMB with the indicated concentrations. Total cell lysates were prepared, and analyzed for iNOS or actin protein by immunoblotting (A). Raw 264.7 cells were pre-treated with LMB in the indicated concentrations for $1 \mathrm{~h}$. Cells were then exposed to catalase, an iNOS inducer (27), for $6 \mathrm{~h}$ in the absence or presence of LMB with the indicated concentrations. Total cell lysates were prepared, and analyzed for iNOS or actin protein by immunoblotting (B).

was sufficient to almost completely down-regulate iNOS expression by LPS in Raw 264.7 cells (Fig. 5A, lane 5). Recently, we have reported that iNOS expression is highly increased by the exposure of catalase, an antioxidant enzyme, into Raw 264.7 cells (9). This led us to further test if LMB inhibits iNOS expression by catalase. As shown in Fig. 5B, LMB potently inhibited the expression of iNOS by catalase in Raw 264.7 cells. Together, these data strongly indicate that LMB inhibition of iNOS expression is not limited to LPS and BV2 cells.

\section{Discussion}

Excessive iNOS expression is associated with a variety of brain diseases, such as neuronal inflammation. Any compound that affects iNOS expression has the potential to be clinically useful against iNOS-associated neuronal diseases. In this study, we have evaluated the anti-inflammatory effect of LMB, an inhibitor of CRM1 nuclear export receptor, on the context of regulation of iNOS expression in response to LPS in BV2 microglial cells. Here we demonstrate, for the first time, that LMB potently inhibits LPS-induced iNOS expression in BV2 cells and the inhibition is, in part at least, associated with the ability of LMB to inhibit iNOS mRNA nuclear export, mRNA stability, and transcription.

It has been reported that the primary cellular target of LMB is CRM1, which mediates delivery of cellular RNAs or proteins containing NES (22). Supporting this, recent studies have shown that LMB interferes with nuclear export of RNAs, such as c-fos (24) or COX-2 (27), and of NES-containing proteins, such as $\alpha$-catenin (25) or IкB- $\alpha$ (26). Given that CRM1 mediates nuclear trafficking of cellular RNAs and/or proteins and LMB inactivates CRM1, it is believed that LMB may be a useful experimental tool to understand regulation of gene expression in the context of RNA nuclear export. Timely nuclear export of RNA upon transcription into the cytoplasm where translation occurs is critical for gene expression (28). Presently, there is no report as to the relationship between LMB and iNOS mRNA nuclear export as well as iNOS expression. In this study, through RNA fractionation experiments, we have demonstrated that LPS induces cytoplasmic localization of iNOS mRNA in BV2 cells, but LMB inhibits the process, rather causing nuclear retention of iNOS mRNA. Given that LMB is an inhibitor of CRM1 (22), it is likely that iNOS mRNA nuclear export induced by LPS in BV2 cells is CRM1-dependent. To our knowledge, this is the first report showing LMB regulation of iNOS expression at level of iNOS mRNA nuclear export, a novel post-transcriptional regulation of iNOS expression by LMB. It has been shown that LMB inhibition of CRM1mediated export pathway affects the expression levels of $<2 \%$ of detectable mRNAs in Drosophila Schneider (S2) cells (29). Thus, our present data showing that LMB did not affect nuclear export and subcellular distribution of GAPDH mRNA in response to LPS in BV2 cells may suggest the specificity of LMB to block iNOS mRNA nuclear export in response to LPS in BV2 cells.

Besides the LMB inhibitory effect on iNOS mRNA nuclear export, in this study, we have shown that LMB partially inhibits iNOS transcription by LPS in BV2 cells. It is well established that iNOS transcription largely depends on the activities of transcription factors, including NF- $\mathrm{NB}$, activator protein-1, NF-IL6, or IFN- $\gamma$ responsive factor, which individually binds its cognate $c i s$-acting elements within the iNOS promoter (11-13). Among these, activation of NF-кB by LPS is linked to proteolytic degradation of IкB- $\alpha$, an inhibitor of NF-кB (30). Interestingly, IкB- $\alpha$ is reported to have NES moieties and thus able to shuttle between the cytoplasm and the nucleus (31). Indeed, it has been demonstrated that LMB causes nuclear retention of IкB- $\alpha$, which protects proinflammatory cytokines IL-1ß- or TNF- $\alpha$-induced degradation

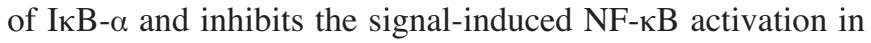
HeLa cells (32). However, in this study, we observed that LMB has no effect on subcellular distribution of IкB- $\alpha$ (data not shown) and LPS-induced degradation of IкB- $\alpha$ in BV2 cells, suggesting that LMB inhibition of LPS-induced iNOS transcription may be unrelated to NF- $\mathrm{KB}$. Thus it is likely that LMB may affect other transcriptional activators or repressors whose activities or nuclear exporting are necessary for iNOS transcription. We also showed that iNOS mRNA induced by LPS in BV2 cells is unstable in the presence of $\mathrm{LMB}$, as determined by actinomycin $\mathrm{D}$ chase experiments. These data suggest that LMB may exert its inhibitory effect on iNOS expression at the levels of transcription and posttranscription such as mRNA stability and nuclear export.

Accordingly, iNOS expression is shown to be strongly related with activation of PI3K, AKT, Ras, Raf, ERKs, p38s, and/or JNKs (17-20,33). Timely localization of some cytoplasmic signaling proteins or transcription factors into the nucleus where gene transcription occurs is a pivotal step 
in the activation of gene expression in response to extracellular signals (34). ERKs, which locate to the cytoplasm in quiescent cells, translocate to the nucleus upon treatment with extracellular stimuli (35). LMB was shown to inhibit the nuclear export of ERKs (35) and MEK-1/2, the upstream activator of ERKs (36). Inconsistent with this, we found that LMB does not affect nuclear localization (data not shown) and activation of ERKs, p38s, or JNKs induced by LPS in BV2 cells. It is, therefore, unlikely that LMB may exert its inhibitory effect on LPS-induced iNOS expression in BV2 cells through modulation of MAPKs.

An interesting result in the present study is that with similar potency LMB can suppress iNOS expression by LPS or catalase, an inducer of iNOS (9), also in Raw 264.7 cells, indicating that LMB inhibition of iNOS expression is not limited to LPS and BV2 cells.

In conclusion, we demonstrate the potency and strength of LMB to inhibit the expression of iNOS induced by LPS in BV2 microglia. LMB may exert its inhibitory effect on LPS-induced iNOS expression in BV2 microglia, at least in part, by down-regulating iNOS transcription, reducing iNOS mRNA stability, and interfering with iNOS mRNA nuclear export. Future strategies are warranted to better apply LMB (or its analogues) as a potent anti-inflammatory agent against neuronal diseases where deregulated expression of inflammatory enzymes such as iNOS is problematic.

\section{Acknowledgements}

We thank Dr C.J. Lowenstein (Johns Hopkins University, MD) for kindly providing us a luciferase DNA construct containing a murine iNOS promoter. This work was supported by Grant No. R13-2002-028-01001-0 from the Basic Research Program of the Korea Science \& Engineering Foundation (KOSEF) to Chronic Disease Research (CDR) Center at Keimyung University.

\section{References}

1. Moncada S and Higgs EA: Endogenous nitric oxide: physiology, pathology and clinical relevance. Eur J Clin Invest 21: 361-374, 1991.

2. Maeda $\mathrm{H}$ and Akaike T: Nitric oxide and oxygen radicals in infection, inflammation, and cancer. Biochemistry 63: 854-865, 1998.

3. Liu CY, Wang CH, Chen TC, Lin HC, Yu CT and Kuo HP: Increased level of exhaled nitric oxide and up-regulation of inducible nitric oxide synthase in patients with primary lung cancer. Br J Cancer 78: 534-541, 1998.

4. Nathan C and Xie QW: Nitric oxide synthases: roles, tolls, and controls. Cell 78: 915-918, 1994.

5. Nathan $\mathrm{C}$ and Xie QW: Regulation of biosynthesis of nitric oxide. J Biol Chem 269: 13725-13728, 1994.

6. Knowles RG and Moncada S: Nitric oxide synthases in mammals. Biochem J 298: 249-258, 1994.

7. Xie QW, Cho HJ, Calaycay J, Mumford RA, Swiderek KM, Lee TD, Ding A, Troso T and Nathan C: Cloning and characterization of inducible nitric oxide synthase from mouse macrophages. Science 256: 225-228, 1992.

8. Chu SC, Marks-Konczalik J, Wu HP, Banks TC and Moss J: Analysis of the cytokine-stimulated human inducible nitric oxide synthase (iNOS) gene: characterization of differences between human and mouse iNOS promoters. Biochem Biophys Res Commun 248: 871-878, 1998.

9. Jang BC, Paik JH, Kim SP, et al: Catalase induces the expression of inducible nitric oxide synthase through activation of NF-kappaB and PI3K signaling pathway in Raw 264.7 cells. Biochem Pharmacol 68: 2167-2176, 2004.
10. Wang Y and Marsden PA: Nitric oxide synthases: gene structure and regulation. Adv Pharmacol 34: 71-90, 1995.

11. Xie QW, Whisnant R and Nathan C: Promoter of the mouse gene encoding calcium-independent nitric oxide synthase confers inducibility by interferon gamma and bacterial lipopolysaccharide. J Exp Med 177: 1779-1784, 1993.

12. Goldring CE, Reveneau S, Algarte $\mathrm{M}$ and Jeannin JF: In vivo footprinting of the mouse inducible nitric oxide synthase gene: inducible protein occupation of numerous sites including Oct and NF-IL6. Nucleic Acids Res 24: 1682-1687, 1996.

13. Dlaska M and Weiss G: Central role of transcription factor NFIL6 for cytokine and iron-mediated regulation of murine inducible nitric oxide synthase expression. J Immunol 162: 6171-6177, 1999

14. Jeohn GH, Chang RC, Kim WG, Wilson B, Mohney RP, Wetsel WC and Hong JS: Post-transcriptional inhibition of lipopolysaccharide-induced expression of inducible nitric oxide synthase by Go6976 in murine microglia. Brain Res Mol Brain Res 79: 18-31, 2000.

15. Lee J, Ryu H, Ferrante RJ, Morris SM and Ratan RR: Translational control of inducible nitric oxide synthase expression by arginine can explain the arginine paradox. Proc Natl Acad Sci USA 100: 4843-4848, 2003.

16. El-Gayar S, Thuring-Nahler H, Pfeilschifter J, Rollinghoff M and Bogdan C: Translational control of inducible nitric oxide synthase by IL-13 and arginine availability in inflammatory macrophages. J Immunol 171: 4561-4568, 2003.

17. Salh B, Wagey R, Marotta A, Tao JS and Pelech S: Activation of phosphatidylinositol 3-kinase, protein kinase B, and p70 S6 kinases in lipopolysaccharide-stimulated Raw 264.7 cells: differential effects of rapamycin, Ly294002, and wortmannin on nitric oxide production. J Immunol 161: 6947-6954, 1998.

18. Paul A, Doherty $\mathrm{K}$ and Plevin R: Differential regulation by protein kinase $\mathrm{C}$ isoforms of nitric oxide synthase induction in RAW 264.7 macrophages and rat aortic smooth muscle cells. Br J Pharmacol 120: 940-946, 1997.

19. Cruz MT, Duarte CB, Goncalo M, Carvalho AP and Lopes MC: Involvement of JAK2 and MAPK on type II nitric oxide synthase expression in skin-derived dendritic cells. Am J Physiol 277: C1050-C1057, 1999.

20. Bhat NR, Zhang P, Lee JC and Hogan EL: Extracellular signalregulated kinase and p38 subgroups of mitogen-activated protein kinases regulate inducible nitric oxide synthase and tumor necrosis factor-alpha gene expression in endotoxinstimulated primary glial cultures. J Neurosci 18: 1633-1641, 1998.

21. Hamamoto T, Gunji S, Tsuji H and Beppu T: Leptomycins A and $\mathrm{B}$, new antifungal antibiotics. I. Taxonomy of the producing strain and their fermentation, purification and characterization. J Antibiot 36: 39-45, 1983.

22. Nishi K, Yoshida M, Fujiwara D, Nishikawa M, Horinouchi S and Beppu T: Leptomycin B targets a regulatory cascade of crm1, a fission yeast nuclear protein, involved in control of higher order chromosome structure and gene expression. J Biol Chem 269: 6320-6324, 1994.

23. Fornerod M, Ohno M, Yoshida M and Mattaj IW: CRM1 is an export receptor for leucine-rich nuclear export signals. Cell 90: 1051-1060, 1997.

24. Brennan CM, Gallouzi IE and Steitz JA: Protein ligands to HuR modulate its interaction with target mRNAs in vivo. J Cell Biol 151: 1-14, 2000.

25. Giannini A, Mazor M, Orme M, Vivanco M, Waxman J and Kypta R: Nuclear export of alpha-catenin: overlap between nuclear export signal sequences and the beta-catenin binding site. Exp Cell Res 295: 150-160, 2004.

26. Huang TT, Kudo N, Yoshida M and Miyamoto S: A nuclear export signal in the N-terminal regulatory domain of IkappaBalpha controls cytoplasmic localization of inactive NF-kappaB/ IkappaBalpha complexes. Proc Natl Acad Sci USA 97: 1014-1019, 2000.

27. Jang BC, Munoz-Najar U, Paik JH, Claffey K, Yoshida M and Hla T: Leptomycin B, an inhibitor of the nuclear export receptor CRM1, inhibits COX-2 expression. J Biol Chem 278: 2773-2776, 2003.

28. Erkmann JA and Kutay U: Nuclear export of mRNA: from the site of transcription to the cytoplasm. Exp Cell Res 296: 12-20, 2004.

29. Herold A, Teixeira L and Izaurralde E: Genome-wide analysis of nuclear mRNA export pathways in Drosophila. EMBO J 22: 2472-2483, 2003. 
30. Simeonidis S, Stauber D, Chen G, Hendrickson WA and Thanos D: Mechanisms by which IkappaB proteins control NF-kappaB activity. Proc Natl Acad Sci USA 96: 49-54, 1999.

31. Lee SH and Hannink M: Characterization of the nuclear import and export functions of Ikappa B (epsilon). J Biol Chem 277: 23358-23366, 2002.

32. Rodriguez MS, Thompson J, Hay RT and Dargemont C: Nuclear retention of IkappaBalpha protects it from signal-induced degradation and inhibits nuclear factor kappaB transcriptional activation. J Biol Chem 274: 9108-9115, 1999.

33. Zhang B, Perpetua M, Fulmer M and Harbrecht BG: JNK signaling involved in the effects of cyclic AMP on IL-1beta plus IFN gamma-induced inducible nitric oxide synthase expression in hepatocytes. Cell Signal 16: 837-846, 2004.
34. Yashiroda Y and Yoshida M: Nucleo-cytoplasmic transport of proteins as a target for therapeutic drugs. Curr Med Chem 10: 741-748, 2003.

35. Adachi M, Fukuda M and Nishida E: Nuclear export of MAP kinase (ERK) involves a MAP kinase kinase (MEK)-dependent active transport mechanism. J Cell Biol 148: 849-856, 2000.

36. Yao Z, Flash I, Raviv Z, Yung Y, Asscher Y, Pleban S and Seger R: Non-regulated and stimulated mechanisms cooperate in the nuclear accumulation of MEK1. Oncogene 20: 75887596, 2001. 